\title{
A COST EFFECTIVE DVI INTERFACE ON VIRTEX 5 FPGA THROUGH VERILOG HDL
}

\author{
Asif Ahmad A S \\ ELECTRONICS AND COMMUNICATION ENGINEERING DEPARTMENT \\ USN:1BM12EC022 \\ BMS COLLEGE OF ENGINEERING,ACCREDITED BY NBA \\ BANGALORE,INDIA \\ asif949@gmail.com
}

\section{ABSTRACT}

There is a definite need for video and image processing technologies in today's world. However the computer vision technologies need to be tested and optimized. There is need for testing these interfaces for the platform which we work on. This modeling is a cost effective architecture for interfacing Digital Visual Interface(DVI) on Virtex5 FPGA's. The architecture is modeled in such a way that it does not use XPS micro blaze or Power PC processor but simple pixel feeder design, configuration of the Chrontel 7301C chip design and the interface between them.

\section{Indexing terms/Keywords}

PixelFeeder, DVI, pixel counter, SDR2DDR, I2CDRAM master, FIFO initial, PLL.

\section{Academic Discipline And Sub-Disciplines}

Computing sciences, Electrical Engineering, VLSI/FPGA Image Processing.

\section{SUBJECT CLASSIFICATION}

VLSI design and Embedded Systems, Electrical and Computing Sciences

TYPE (METHOD/APPROACH)

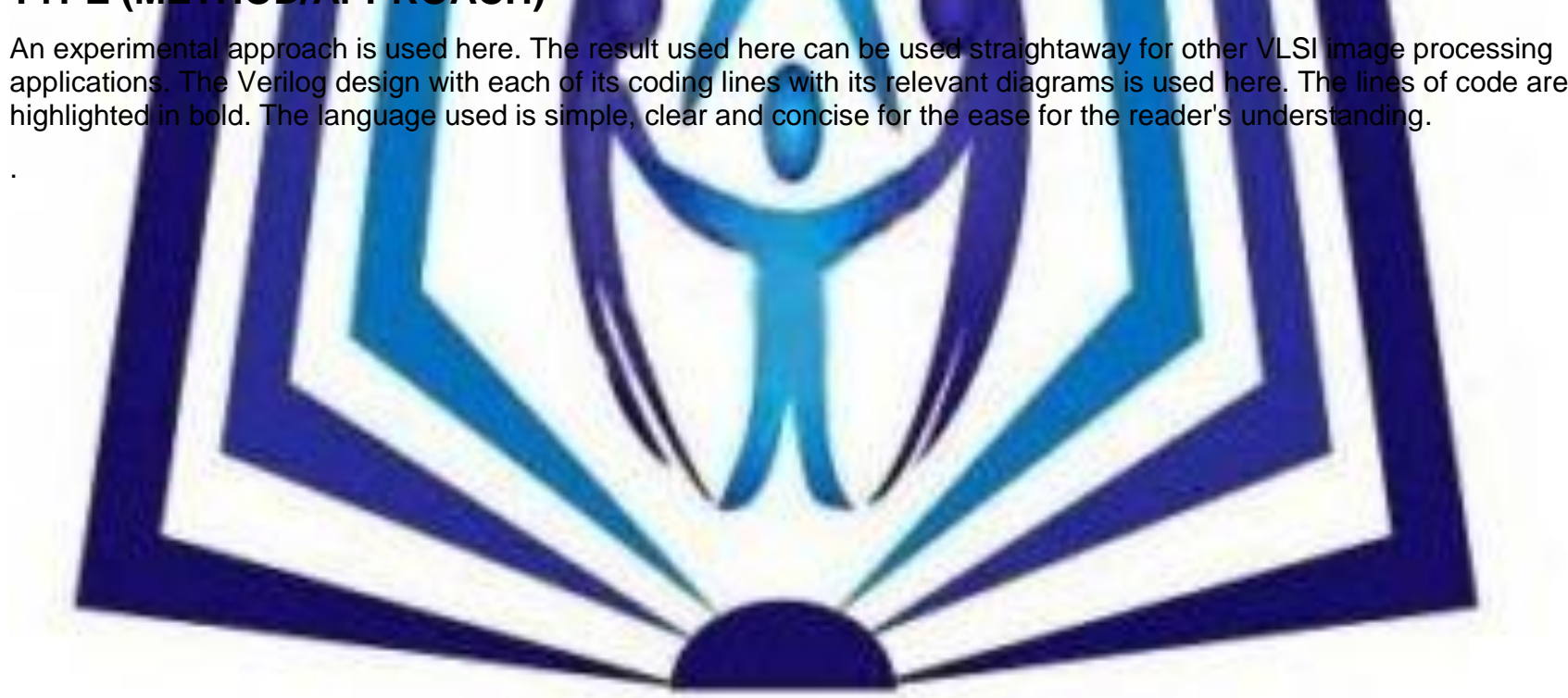

\section{Council for Innovative Research}

Peer Review Research Publishing System

Journal: INTERNATIONAL JOURNAL OF COMPUTERS \& TECHNOLOGY

Vol 13, No. 2

editor@cirworld.com

$\underline{\text { www.cirworld.com, www.ijctonline.com }}$ 


\section{INTRODUCTION}

The design of the DVI interface on virtex 5 requires the modeling of a pixel feeder module design and the correct DVI interfaces between them. The DVI chip present on the FPGA is Chrontel 7301C. The CH7301C is a display controller chip which accepts a digital input signal, encodes it and transmits data through a DVI(Digital Visual Interface or a DFP(Digital flat panel). The DVI module handles communication with the Chrontel DVI chip and initializes its control registers via an I2C bus to set the desired color format and update the pixel rate. Thus the DVI driver needs a constant stream supply of pixels to initialize the clock rate and to output the pixel. Thus a pixel feeder module is necessary. The DVI driver causes the Chrontel DVI chip to generate Synchronous VGA $800 \times 600$ at $75 \mathrm{hz}$. The pixel feeder module is responsible for continuously reading from the frame buffer of the SDRAM and feeding the pixel values to the DVI driver module.

The aim of this paper is to design a fully customized design or a DVI interface by Verilog HDL. This is a cost effective algorithm as it does not require the inclusion of external libraries of XPS and Xilinx EDK. The use of these software forces the inclusion of a MICROBLAZE or a POWER PC soft core processor which gives an unnecessary load for including xps_tft, input output modules such as GPIO,UART, bus peripherals. Another disadvantage of these software is that these IP's cost a lot. Even after addition of these IP's the test software(.elf files) IP's to run on these soft core processors also has to be bought. Also running a software on a processor reduces its speed of execution unless and until there is software parallelization. Amdahl's law states that the maximum speed up of the parallelized version is 1.136 times as fast as the non-parallelized implementation[courtesy-Wikipedia].

Thus this type of approach is money-spinning and not efficient. My algorithm is to completely model a hardware architecture on the FPGA that increases its performance and reduce its cost when used for high speed image processing, video processing and computer vision applications.
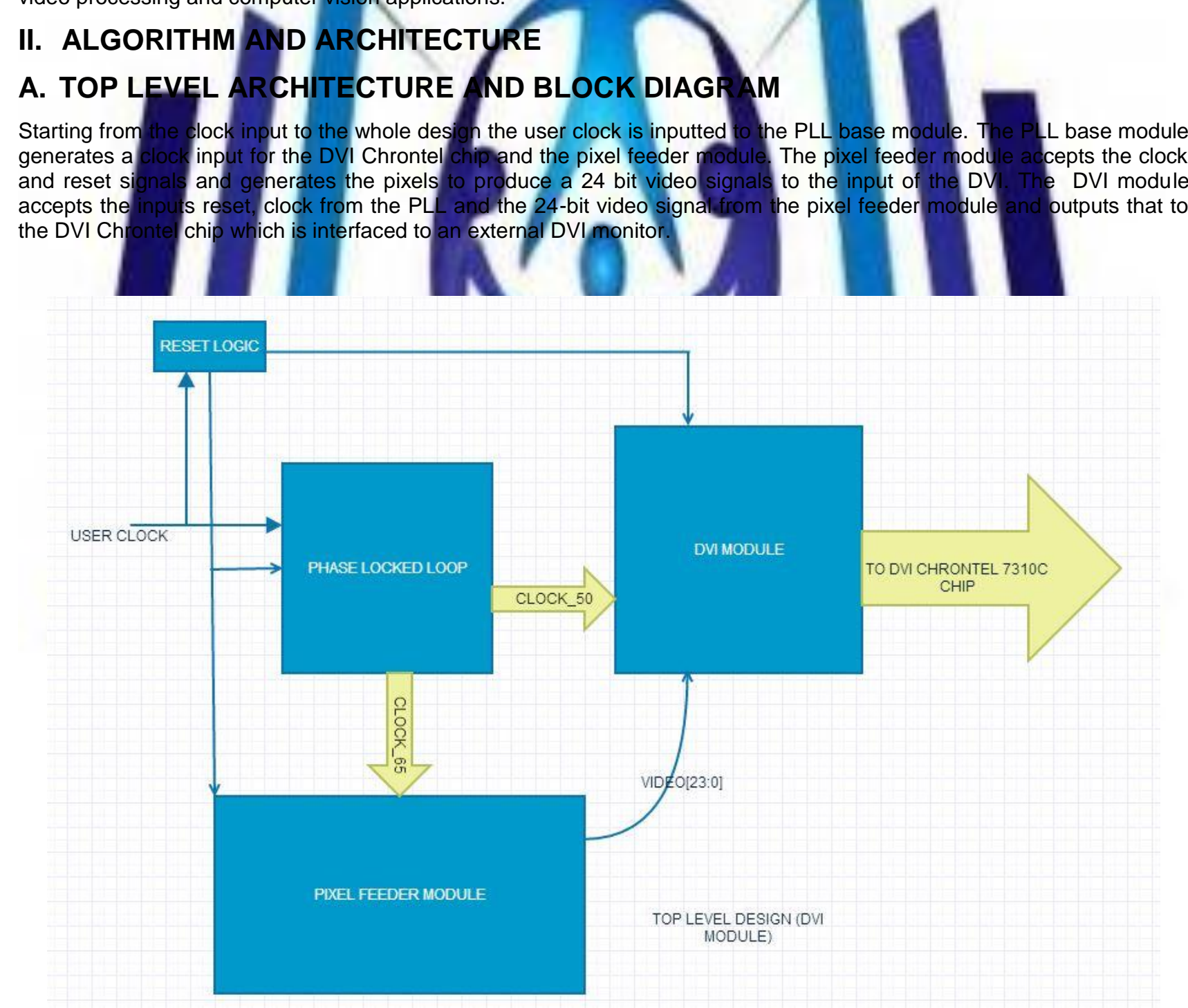


\section{B. PLL_BASE MODULE}

The Chrontel chip needs an external clock supply for active functioning. The PLL(Phase Locked Loop) clock jitter module can eliminate this need. The user clock is input from one of the crystal oscillators on the board and is multiplied by this module. The output from this module consists of a series of clock frequencies which can be input to the pixel feeder and the DVI modules. Below are some lines of code.

This code is used to output certain clock frequencies out from the PLL module.

IBUFG user_clk_buf (.I(USER_CLK), .O(user_clk_g) ); BUFG cpu_clk_buf ( .I(cpu_clk), .O(cpu_clk_g) );

BUFG clk200_buf (.I(clk200), .O(clk200_g) );

BUFG clk0_buf (.I(clk0), .O(clk0_g) );

BUFG clkdiv50_buf (.l(clk50), .O(clk50_g) );

BUFG clk90_buf (.I(clk90), .O(clk90_g) );

BUFG clkdiv0_buf (.l(clkdiv0), .O(clkdiv0_g) ));

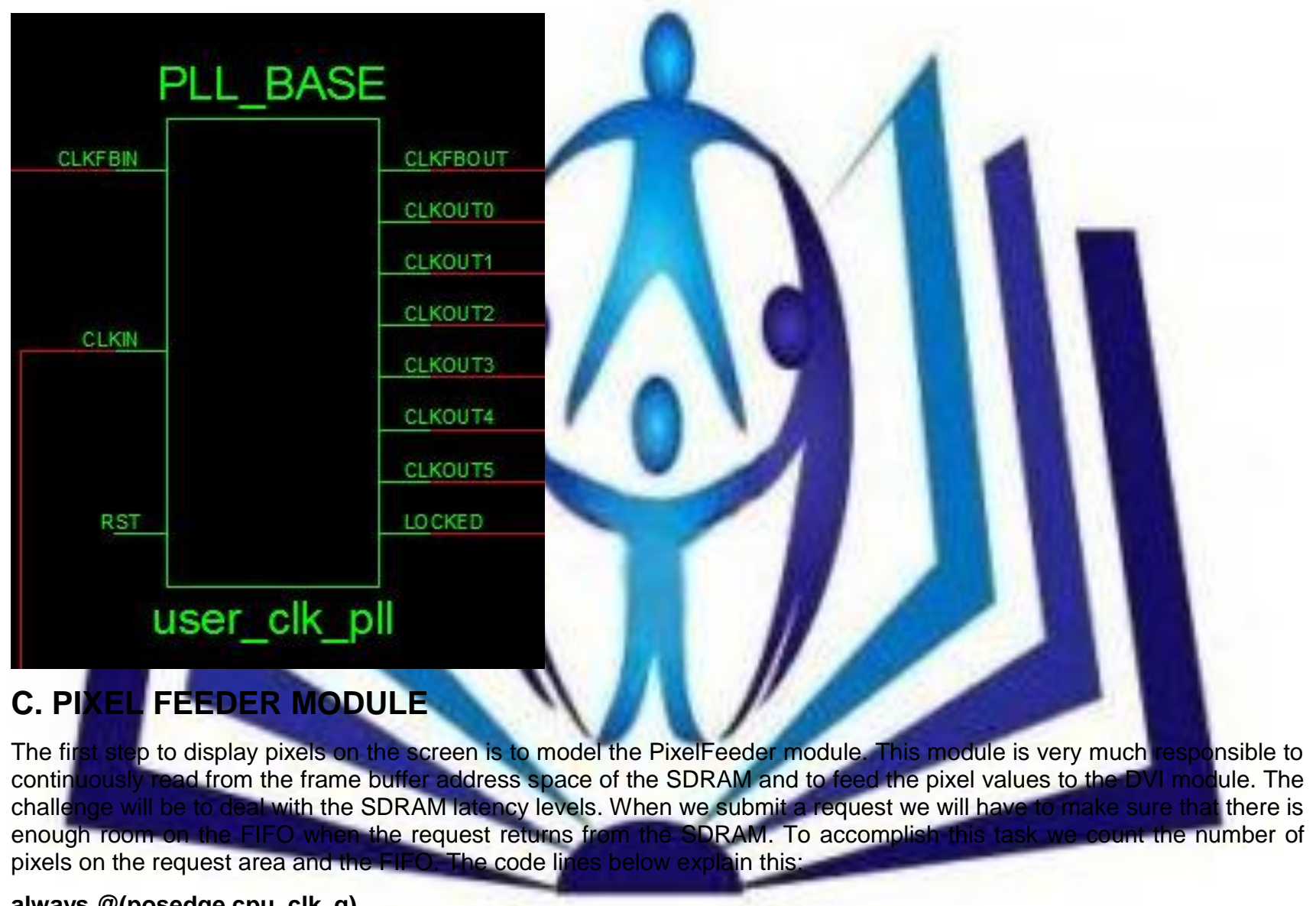

always @(posedge cpu_clk_g)

begin

$$
\text { if(!feeder_full) }
$$

begin

valid <= 1;

dout <=128'hffffffffffffffffffffffffffff; //rdf_dout; //read in processed output pixels

end

else begin

valid <= 0;

end

end 


\begin{abstract}
//assign dout = 128'hfffffffffffffffffffffffffff;
//assign valid = 1'b1;
\end{abstract}

always @(*) begin

if (video_ready) begin

video = feeder_dout[23:0];

video_valid = 1'b1;

end

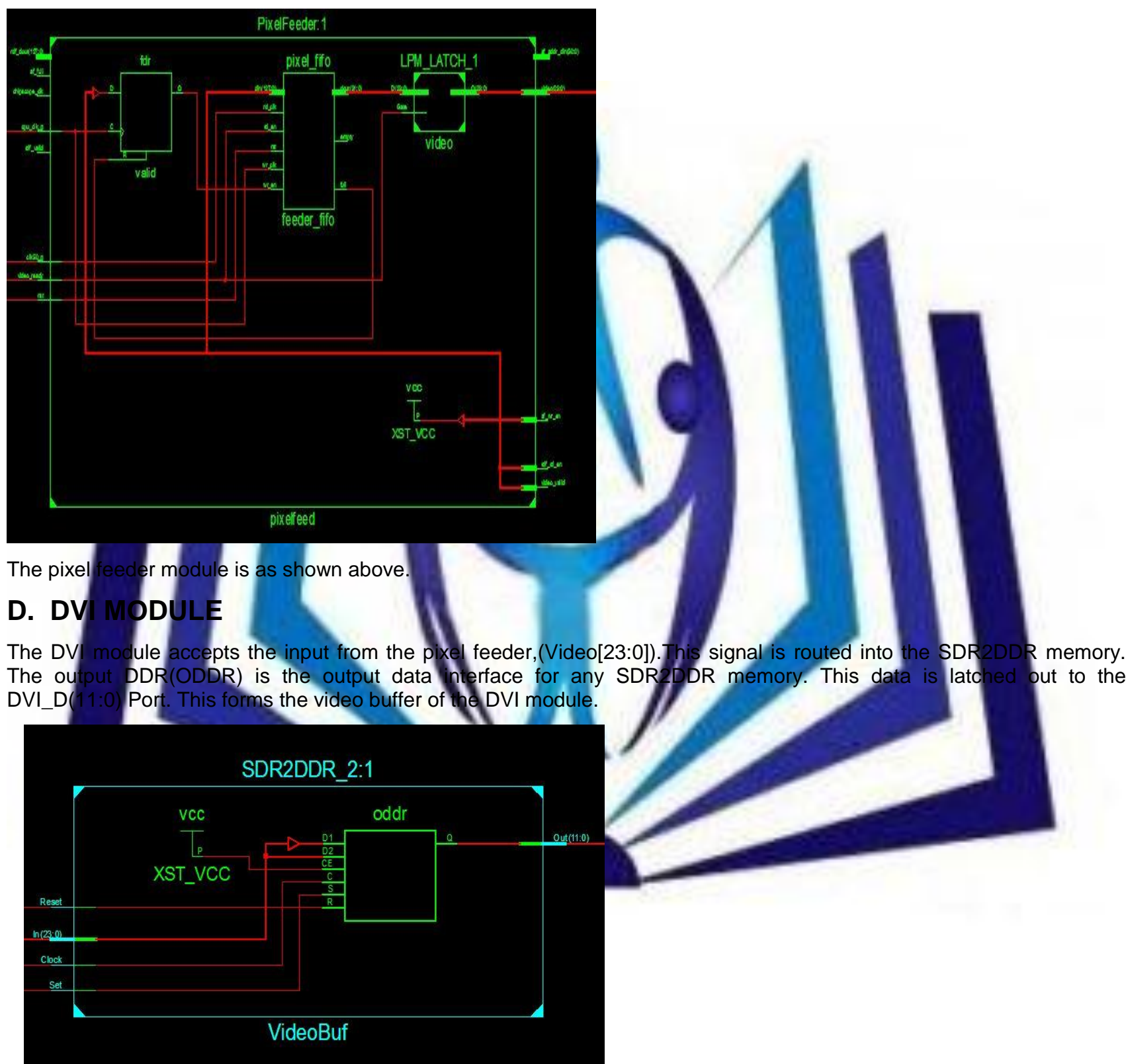

For Hsync and Vsync of the DVI interface display, an IORegister module has a simple D flip flop to and produces a DVI_H,DVI_V,DVI_D signals. The pixel active and the video ready signals must be fed back into the pixel feeder FIFO so that the pixel feeder must output a next pixel. As said above the challenge in this kind of design is to maintain and deal with the SDRAM latency when the above operation is accomplished. The best way to accomplish this task is to count the number of pixels on the request area and the FIFO. The videoready signal is fed back to the pixel feeder module through the pixel counter. Thus the pixel counter counts the number of pixels in the request area and is fed back to the LPM_LATCH in the pixel feeder. The pixel must be count in both the $X$ and $Y$ directions, stored in a register and must be compared with the request area. Similarly the Hsync and Vsync signals are compared and stored. Thus at the end of a pixel write onto the DVI chip, the SDRAM pulls out an interrupt and feeds it back into the pixel FIFO to know it is full. The 
module below shows the count request area for the X-direction. The register is a behavioral register whose code is as shown below.
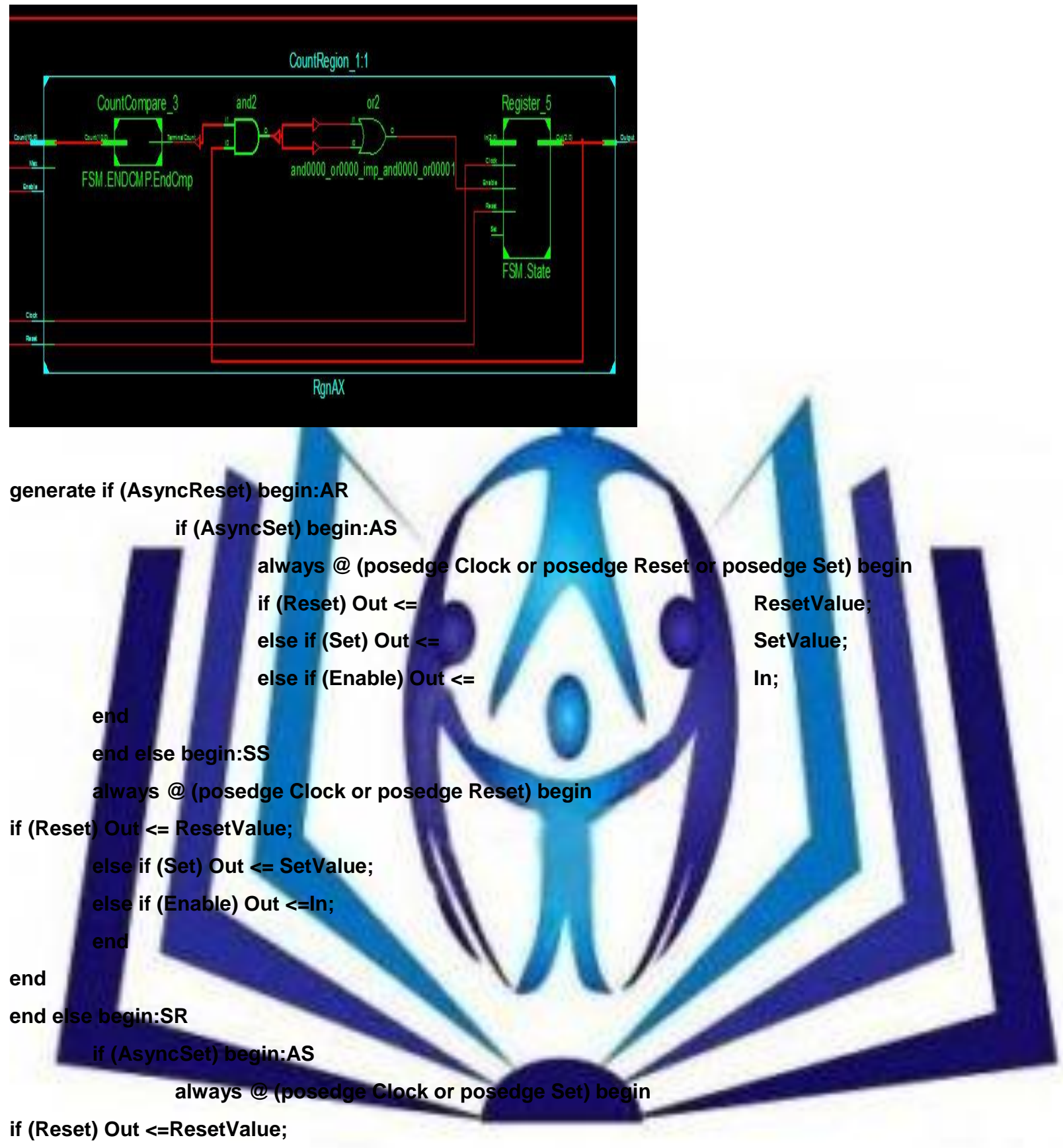

else if (Set) Out <=SetValue;

else if (Enable) Out $<=$ In;

end

end

else begin:SS

always@ @ (posedge Clock) begin

if (Reset) Out <=ResetValue;

else if (Set) Out <= SetValue;

else if (Enable) Out <=In;

end 
end

\section{end endgenerate}

Similarly the Y-direction is checked and thus the Hsync and Vsync signals.

Thus the interface is established to the DVI display controller.

\section{RESULTS}

The best results were obtained and the output was checked on a DVI monitor. The interface gave an output of a white screen on the DVI display. The display was also used for checking the changing inverting patterns and was also successful. Thus DVI interface chip was successfully modeled. The applications was also modeled for video processing which still gave me the best results.

\section{APPLICATIONS}

The interface can be directly used for interfacing video processing, image processing algorithms. The algorithm module must be connected to the inputs of the pixel feeder circuit. The video interface can be input into a VGA input video codec, the pixels must be processed, and fed into the pixel feeder again and then output to the DVI module where the pixel is output onto the screen. Thus this low cost algorithm is very efficient in performance and speed for video processing applications and can be used even without the XPS or EDK software.

\section{CONCLUSION}

The DVI interface was a proposed base work to establish an easy way to interface core image processing and video processing projects and was completed successfully. I have also checked the system with some interfacing of video and image algorithms such as stream - videfrrion on an stream - viden processing, all of which gave $m$ e high quality results and this method of DVI interface on an FPGA can boost the engineer's knov processing and segmentation and also in the field of video processing ant plans of this project are to take this algorithm onto computer vision app replaced on a hardware accelerator or an interface chip and all the pre also doubles the speed of the CPU, increases power efficiency, and also is of resources is established. I guarantee that this work can be readily used application

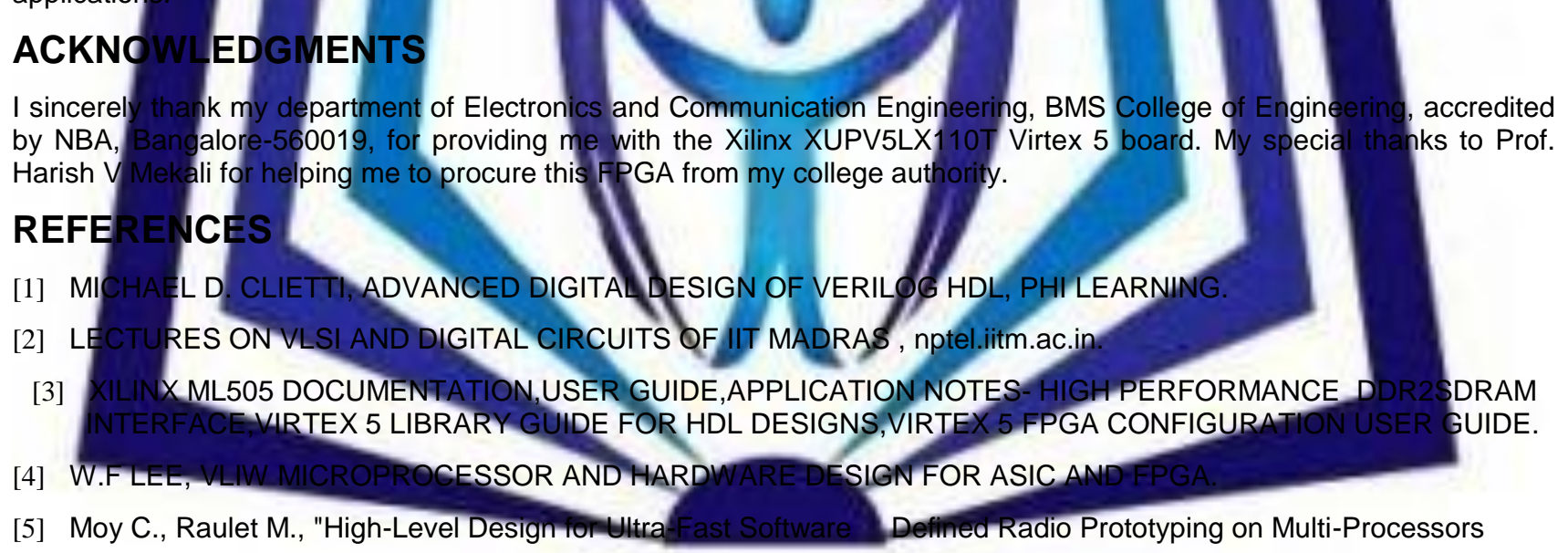

Heterogeneous Platforms", Journal on Advances in Electronics and Telecommunications - Radio Communication Series: special issue on Recent Advances and Future Trends in Wireless (Communications, Vol. 1, $\mathrm{n}^{\circ} 1, \mathrm{pp} .67-85$, April 2010[in press].)

[6] PONG.P.CHU, FPGA PROTOTYPING BY VERILOG EXAMPLES.

[7] CLASS LECTURES OF Prof. AJAY D KUMAR ON VHDL AND VERILOG DESCRIPTION LANGUAGES, BMS COLLEGE OF ENGINEERING, ACCREDITED BY NBA, BANGALORE-560078. 


\section{Author' biography}

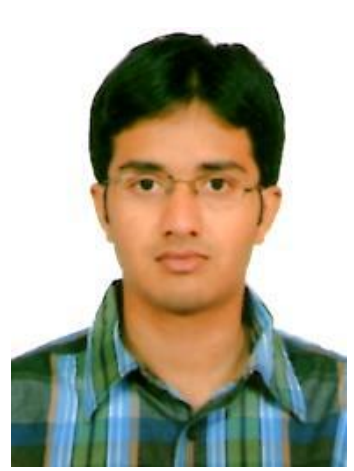

Asif Ahmad A S currently pursuing his 2nd year engineering degree in Electronics and Communication Engineering,BMSCE, accredited by NBA,NAAC(A grade),BANGALORE,INDIA. $\mathrm{He}$ has been the member of NXP laboratories,BMSCE(Council Of Innovation and Reasearch) has worked on "smart low power irrigation in remorte areas" which bagged 2nd place in ARM technical symposium. He also earned a "Certificate of Merit" for the national level robotics championship held in INDIA.

His interests are VLSI design and architectures for signal processing algorithms, efficient algorithms for signal processing filter designs, etc. Now he has been working on Efficient VLSI Architectures for image impainting, filter designs. He has worked on many projects such as "Sleep low power modes of ARM Cortex M0,M3",GSM communication and data transfer", "SPI protocol between GSM and ARM Cortex M4", etc. He has a wider vision of application and confines his works to a research degree.

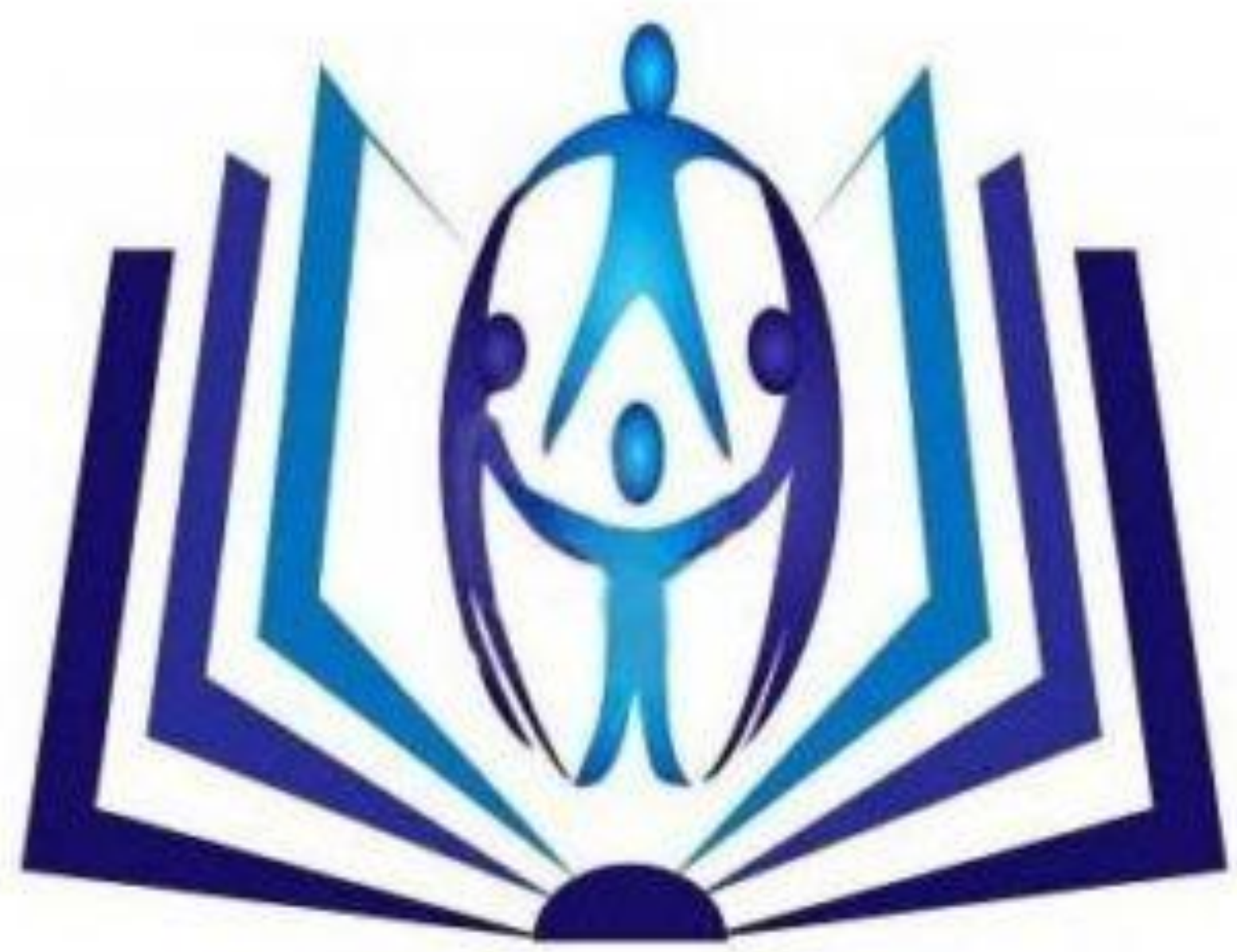

